\title{
A RIGID SPACE $X$ FOR WHICH $X \times X$ IS HOMOGENEOUS; AN APPLICATION OF INFINITE-DIMENSIONAL TOPOLOGY
}

JAN VAN MILL

\begin{abstract}
We give an example of a rigid (= no autohomeomorphisms beyond the identity) space $X$ such that $X \times X$ is homogeneous. In fact, $X \times X$ is homeomorphic to the Hilbert cube. This answers a question of A. V. Arhangel'skii.
\end{abstract}

1. Introduction. In his survey paper [1, p. 59], A. V. Arhangel'skii asks whether there is an example of a nonhomogeneous (compact) space whose square is homogeneous. The aim of this note is to show that recent results in infinite-dimensional topology can be used to construct a rigid (= no autohomeomorphisms beyond the identity) space $X$ such that $X \times X \approx Q$, the Hilbert cube. As a consequence, $X \times X$ is homogeneous. Modulo some very nontrivial known facts it is not difficult to construct $X$. Since $X$ will prevent general topologists from asking certain natural questions concerning homogeneity, we feel that our example should appear in print.

For all undefined terms concerning infinite-dimensional topology, see [2]. I am indebted to Jeroen Bruyning and to Marcel van de Vel for some helpful comments.

2. Embedding $M \times Q \times[0,1)$ in $Q$. Wong [12] has shown that wild Cantor sets in $Q$ exist. In [4] Daverman obtained, among others, the same result by totally different methods. We will apply Daverman's construction to obtain an embedding theorem for certain $Q$-manifolds in $Q$.

2.1. TheOREM. Let $M$ be a compact acyclic, $P L$ n-manifold (with boundary). Then there is a finite-dimensional compact $A R B \subset Q$ such that $Q-B \approx M \times Q \times$ $[0,1)$.

Proof. Let $X \subset M$ be an $(n-1)$-spine for $M$ and fix a Cantor set $C$ in the interior of the 2-cell $I^{2}$ and take $q \in Q$ arbitrarily. Daverman [4] shows that $Q^{*}=\left(M \times I^{2} \times Q\right) / G$, where $G$ is the upper semicontinuous decomposition of $M \times I^{2} \times Q$ having $\{X \times\{c\} \times\{q\}: c \in C\}$ for its set of nondegenerate elements, is homeomorphic to $Q$. Let $\pi: M \times I^{2} \times Q \rightarrow Q^{*}$ be the decomposition map. Put $B=\pi\left(M \times I^{2} \times\{q\}\right)$. By [3], $B$ is an AR which, by the countable sum

Received by the editors January 22, 1981.

1980 Mathematics Subject Classification. Primary 54G20; Secondary 52B05. 
theorem, is finite-dimensional. Clearly, $Q^{*}-B=\pi\left(M \times I^{2} \times(Q-\{q\})\right) \approx M$ $\times I^{2} \times(Q-\{q\}) \approx M \times I^{2} \times Q \times[0,1) \approx M \times Q \times[0,1)$. (This proof can be simplified of course. Daverman's construction proves more than we need.)

We will now show that the AR of Theorem 2.1 can be chosen to be of arbitrarily small diameter.

2.2. Lemma. Let $A \subset Q$ be closed and nowhere dense, and let $Z \subset Q$ be a Z-set. Then there is a homeomorphism $h: Q \rightarrow Q$ such that $Z \cap h(A)=\varnothing$.

Proof. Since $A \subset Q$ is nowhere dense, there is a $Z$-set $Z^{\prime} \subset Q-(A \cup Z)$ which is homeomorphic to $Z$. Let $f: Z^{\prime} \rightarrow Z$ be an arbitrary homeomorphism. By the Homeomorphism Extension Theorem [2], there exists a homeomorphism $h: Q$ $\rightarrow Q$ extending $f$. It is clear that $h$ is as required.

2.3. Corollary. Let $M$ be a compact acyclic, PL n-manifold (with boundary) and let $U \subset Q$ be open and nonempty. Then there is a finite-dimensional compact $A R$ $B \subset U$ such that $Q-B \approx M \times Q \times[0,1)$.

Proof. Find an $n \geqslant 1$ and nondegenerate intervals $\left[a_{i}, b_{i}\right] \subset I(i<n)$ such that

$$
Q^{\prime}=\left[a_{1}, b_{1}\right] \times \cdots \times\left[a_{n}, b_{n}\right] \times I \times I \times \cdots \subset U .
$$

Let $S=\left\{x \in Q^{\prime}: \exists i \leqslant n\right.$ with $\left.x_{i} \in\left\{a_{i}, b_{i}\right\}\right\}$. It is clear that $S$ is a $Z$-set in the Hilbert cube $Q^{\prime}$. By Theorem 2.1 and Lemma 2.2 we can find a finite-dimensional compact $\mathrm{AR} B \subset Q^{\prime}-S$ such that $Q^{\prime}-B \approx M \times Q \times[0,1)$. Clearly, $Q-B$ $\approx Q^{\prime}-B$.

3. The example. In this section, $M$ denotes a fixed compact, acyclic, nonsimply connected PL $n$-manifold (with boundary). Corollary 2.3 implies that we can find a family $\left\{K_{i}: i \in \mathbf{N}\right\}$ of finite-dimensional compact AR's in $Q$ such that

(1) $i<j \rightarrow K_{i} \cap K_{j}=\varnothing$,

(2) $\operatorname{diam}\left(K_{i}\right)<2^{-i}$,

(3) $\cup_{1}^{\infty} K_{i}$ is dense in $Q$, and

(4) $Q-K_{i} \approx M^{i} \times Q \times[0,1)$.

Let $G$ be the upper semicontinuous decomposition of $Q$ having $\left\{K_{i}: i \in \mathbf{N}\right\}$ for its set of nondegenerate elements and put $X=Q / G$. Let $\pi: Q \rightarrow X$ be the decomposition. We claim that $X$ is as required.

\subsection{LEMMA. $X$ is an $A R$.}

Proof. Since $\pi$ is cell-like and has only countably many nondegenerate pointinverses, this follows from Kozlowski [6].

\subsection{LEMMA. $X$ is rigid.}

Proof. Since $\pi$ is cell-like and since $X$ is an AR, for each $x \in X$ it is true that $X-\{x\}$ and $Q-\pi^{-1}(x)$ have the same homotopy type, Haver [5]. Let $x_{i}$ be the unique point in $\pi\left(K_{i}\right)(i \in N)$. Since $M$ is nonsimply connected and since the 
fundamental group $\pi_{1}(M)$ is finitely generated $\left[9\right.$, p. 141], it follows that $\pi_{1}\left(M^{i}\right) \approx$ $\pi_{1}\left(M^{j}\right)$ if $i \neq j$. Simply observe that

$$
\pi_{1}\left(M^{i}\right) \approx \underbrace{\pi_{1}(M) * \pi_{1}(M) * \cdots * \pi_{1}(M)}_{i \text { times }}
$$

and that, by Gruško's Theorem [8, p. 58], this implies that the minimum number of generators of $\pi_{1}\left(M^{i}\right)$ is equal to $i$ times the minimum number of generators of $\pi_{1}(M)$. Since $\pi_{1}\left(M^{i}\right) \approx \pi_{1}\left(Q-K_{i}\right) \approx \pi_{1}\left(X-\left\{x_{i}\right\}\right)$ for all $i \in \mathrm{N}$, we can therefore conclude that no autohomeomorphism of $X$ can map some $x_{i}$ onto $x_{j}$ for $i \neq j$. Now take $x \in X-\left\{x_{i}: i \in \mathrm{N}\right\}$. Then $\pi_{1}(X-\{x\}) \approx \pi_{1}(Q-\{\mathrm{pt}\})=0$. Therefore, for the same reason, no autohomeomorphism of $X$ can map $x_{i}$ onto a point of $X-\left\{x_{i}: i \in \mathbf{N}\right\}$. We conclude that each autohomeomorphism of $X$ is the identity on the dense set $\left\{x_{i}: i \in \mathbf{N}\right\}$, hence must be the identity.

\subsection{LEMMA. $X \times X \approx Q$.}

Proof. Since $X$ is an AR by Lemma 3.1, and since $\pi$ is a cell-like map having only countably many nondegenerate, finite-dimensional point-inverses, this follows from Toruńczyk [10, Theorem 5].

4. Remarks. Since our example is infinite-dimensional, one naturally wonders whether a finite-dimensional space exists with similar properties. I do not know the answer to this question. In addition, we have seen, as is well known, that one can use connectivity properties to construct rigid spaces. This type of construction does not work in the zero-dimensional case. In fact, it is much more complicated to construct a rigid zero-dimensional space than a rigid continuum. For details, see Kuratowski [7]. Using results of [9] we can construct a homogeneous subset of the real line which is the union of countably many dense rigid subspaces, but we do not know whether this space admits a product structure. I think it would be very interesting to have a rigid zero-dimensional space with a homogeneous square. One is also naturally led to the question whether there is a rigid space $X$ for which $X \times X$ is a topological group. In conclusion let us pose the following problems (all spaces are separable metric):

(1) Is there a finite-dimensional rigid continuum $X$ for which $X \times X$ is homogeneous?

(2) Is there a rigid space $X$ such that $X \times X$ is a topological group? Can $X$ be a continuum? zero-dimensional?

(3) Is there a rigid zero-dimensional space $X$ for which $X \times X$ is homogeneous?

\section{REFERENCES}

1. A. V. Arhangel'skii, Structure and classification of topological spaces and cardinal invariants, Russian Math. Surveys 33 (1978), 33-96.

2. T. A. Chapman, Lectures on Hilbert cube manifolds, CBMS Regional Conf. Series in Math., no. 28, Amer. Math. Soc., Providence, R. I., 1976.

3. R. J. Daverman, Embedding phenomena based upon decomposition theory: Wild Cantor sets satisfying strong homogeneity properties, Proc. Amer. Math. Soc. 75 (1979), 177-182.

4. __ A strongly homogeneous but wildly embedded Cantor set in the Hilbert cube (to appear). 
5. W. E. Haver, Mappings between ANR's that are fine homotopy equivalences, Pacific J. Math. 58 (1975), 457-461.

6. G. Kozlowski, Images of ANR's, Trans. Amer. Math. Soc. (to appear).

7. K. Kuratowski, Sur la puissance de l'ensembele des "nombres de dimension" de M. Fréchet, Fund. Math. 8 (1925), 201-208.

8. A. G. Kurosh, The theory of groups. II, Chelsea, New York, 1960.

9. J. van Mill, Homogeneous subsets of the real line (to appear).

10. E. H. Spanier, Algebraic topology, McGraw-Hill, New York, 1966.

11. H. Torunczyk, On CE-images of the Hilbert cube and characterization of $Q$-manifolds, Fund. Math. 106 (1980), 31-40.

12. R. Y. T. Wong, A wild Cantor set in the Hilbert cube, Pacific J. Math. 24 (1968), 189-193.

Subfaculteit Wiskunde, VRue Universitert, De Boelelaan 1081, Amsterdam, The Netherlands 